\title{
ON A SINGULAR VALUE INEQUALITY OF KY FAN AND HOFFMAN
}

\author{
PETER G. DODDS AND THERESA K.-Y. DODDS
}

(Communicated by Palle E. T. Jorgensen)

\begin{abstract}
It is shown that the identity operator is a best unitary approximant to any positive measurable operator affiliated with a semifinite von Neumann algebra equipped with a distinguished faithful normal semifinite trace.
\end{abstract}

\section{INTRODUCTION}

It is a well-known result of Fan and Hoffman [FH] that the $n \times n$ identity matrix is a best unitary approximant to any Hermitian positive semidefinite $n \times n$ matrix for every unitarily invariant norm. Extensions of this result to Schatten $p$-classes have been given by [AEG, GK, vR]. In the present paper, we show (Theorem 3.1) that if $x$ is a positive operator on a Hilbert space, measurable with respect to some semifinite von Neumann algebra $\mathscr{M}$ equipped with a distinguished semifinite trace, then the generalized singular value function (or decreasing rearrangement) of $x-1$ is submajorized (in the sense of Hardy, Littlewood, and Polya) by that of $x-u$ for each unitary $u \in \mathscr{M}$. In fact, in the case of $n \times n$ matrices, it is precisely this submajorization result that is proved in $[\mathrm{FH}]$, to which the stated metric inequalities are equivalent. The method of $[\mathrm{FH}]$ is based on a submajorization inequality for the eigenvalues of selfadjoint matrices due to Lidskii [Li] and Wielandt [Wi], subsequently generalized to compact operators by Markus [Ma]. Similarly, the approach of [vR] is based on a very special extension of the Markus inequality to the setting of bounded operators on a Hilbert space, using the generalized notion of singular value for bounded selfadjoint operators that may be found in [GK]. Accordingly, a principal tool on which the present paper is based is a very general form of the Markus inequality for measurable operators that may be found in [DDP2].

In $\S 1$, we gather the relevant terminology and essential properties of generalized singular values of measurable operators that form the basis of our approach, with the principal submajorization result being given in $\S 3$. Since majorization inequalities imply corresponding metric inequalities for fully symmetric operator spaces and since each of the noncommutative $L^{p}$-spaces associated with a

Received by the editors January 21, 1991 and, in revised form, May 14, 1991.

1980 Mathematics Subject Classification (1985 Revision). Primary 46E30; Secondary 46L50, 47D40.

The second author's research was supported by A.R.C. 
semifinite von Neumann algebra is fully symmetric, we recover as special cases of our main result the corresponding metric inequalities given in [vR, AEG, $\mathrm{GK}$ ]. In the final section, we show that if a strictly positive operator admits a best unitary approximant in a noncommutative $L^{p}$-space, with $1<p<\infty$, then this best approximant is necessarily unique. This extends known results for the Schatten $p$-classes due to $[\mathrm{AEG}]$ to the general setting.

\section{Preliminaries}

We denote by $\mathscr{M}$ a semifinite von Neumann algebra in the Hilbert space $H$ with given normal faithful semifinite trace $\tau$. If $x$ is a densely defined linear selfadjoint operator in $H$ and $x=\int_{(-\infty, \infty)} s d e_{s}^{x}$ is its spectral decomposition, then, for any Borel subset $B \subseteq \mathbb{R}$, we denote by $\chi_{B}(x)$ the corresponding spectral projection $\int_{(-\infty, \infty)} \chi_{B}(\bar{s}) d e_{s}^{x}$. A closed densely defined linear operator $x$ in $H$ affiliated with $\mathscr{M}$ is said to be $\tau$-measurable if and only if there exists a number $s \geq 0$ such that

$$
\tau\left(\chi_{(s, \infty)}(|x|)\right)<\infty
$$

The set of all $\tau$-measurable operators will be denoted by $\widetilde{\mathscr{M}}$. The set $\widetilde{\mathscr{M}}$ is $a^{*}$-algebra with sum and product being the respective closures of the algebraic sum and product [Ne]. For $x \in \widetilde{\mathscr{M}}$, the generalized singular value function (or decreasing rearrangement) $\mu .(x)$ of $x$ is defined by

$$
\mu_{t}(x)=\inf \left\{s \geq 0: \tau\left(\chi_{(s, \infty)}(|x|)\right) \leq t\right\}, \quad t \geq 0 .
$$

It follows that $\mu(x)$ is a decreasing, right continuous function on the halfline $\mathbb{R}^{+}=[0, \infty)$. Moreover,

$$
\mu(x y z) \leq\|x\|_{\infty} \mu(y)\|z\|_{\infty}, \quad x, z \in \mathscr{M}, y \in \widetilde{\mathscr{M}}
$$

where $\|.\|_{\infty}$ is the usual operator norm and

$$
\mu(f(|x|))=f(\mu(x))
$$

for any continuous increasing function $f$ on $[0, \infty)$ with $f(0)=0$. For further properties of singular value functions, see [FK].

If $\mathscr{M}$ is the space $L(H)$ of all bounded linear operators on $H$ and $\tau$ is the standard trace, then $\widetilde{\mathscr{M}}=L(H)$ and $x \in L(H)$ is compact if and only if $\mu_{t}(x) \rightarrow 0$ as $t \rightarrow \infty$, in which case for each $n=0,1,2, \ldots$,

$$
\mu_{n}(x)=\mu_{t}(x), \quad t \in[n, n+1),
$$

and $\left\{\mu_{n}(x)\right\}_{n=0}^{\infty}$ is the usual singular value sequence of $x$ in decreasing order counted according to multiplicity [GK].

We identify the space $L^{\infty}\left(\mathbb{R}^{+}\right)$of all bounded complex-valued Lebesgue measurable functions on the halfline $\mathbb{R}^{+}$as a commutative von Neumann algebra acting by multiplication on the Hilbert space $L^{2}\left(\mathbb{R}^{+}\right)$with trace given by integration with respect to Lebesgue measure $m$. In this case, the $\tau$-measurable operators coincide with those complex measurable functions $f$ on $\mathbb{R}^{+}$that are bounded except on a set of finite measure. In this example, the generalized singular value function, which we continue to denote by $\mu(f)$, coincides with the familiar right continuous decreasing rearrangement of the function $f$. See, for example, [KPS]. 
If $x, y \in \widetilde{\mathscr{M}}$, we say that $x$ is submajorized by $y$, written $x \nprec y$, if and only if

$$
\int_{0}^{a} \mu_{t}(x) d t \leq \int_{0}^{a} \mu_{t}(y) d t \quad \text { for all } a \geq 0 .
$$

The following result is proved in [DDP2].

$$
\text { If } x, y \in \widetilde{\mathscr{M}} \text { then } \mu(x)-\mu(y) \nprec \mu(x-y) \text {. }
$$

We remark that the submajorization is, of course, with respect to the von Neumann algebra $L^{\infty}\left(\mathbb{R}^{+}\right)$. This submajorization inequality generalizes a similar inequality for compact operators proved by Markus [Ma], and accordingly we refer to it as the generalized Markus inequality. We note, as a simple consequence, that if $x, y \in \widetilde{\mathscr{M}}$ then

$$
\mu(x+y) \nprec \mu(x)+\mu(y) .
$$

We define

$$
\widetilde{\mathscr{M}_{0}}=\left\{x \in \widetilde{\mathscr{M}}: \mu_{t}(x) \rightarrow 0 \text { as } t \rightarrow \infty\right\} .
$$

It is clear that $x \in \widetilde{\mathscr{M}}_{0}$ if and only if $\tau\left(\chi_{(s, \infty)}(|x|)\right)<\infty$ for all $s>0$.

\section{A Singular VAlUe EQUALITY}

Lemma 2.1. If $\tau(1)<\infty$ and $0 \leq x \in \widetilde{\mathscr{M}}$ then

$$
\mu(x-1)=\mu(\mu(x)-\mu(1)) \text {. }
$$

Proof. It is clear that

$$
\tau\left(\chi_{(s, \infty)}(|x-1|)\right)=\tau\left(\chi_{[0,1-s)}(x)\right)+\tau\left(\chi_{(1+s, \infty)}(x)\right), \quad s \geq 0,
$$

and

$$
\tau\left(\chi_{(s, \infty)}(x)\right)=m\left\{r \geq 0: \mu_{r}(x)>s\right\}, \quad s \geq 0 .
$$

Since $\tau(1)<\infty$, it follows routinely from the normality of $\tau$ that

$$
\tau\left(\chi_{[s, \infty)}(x)\right)=m\left\{r \geq 0: \mu_{r}(x) \geq s\right\}, \quad s \geq 0,
$$

and so

$$
\tau\left(\chi_{[0, s)}(x)\right)=m\left\{r: 0 \leq r \leq \tau(1) \text { and } \mu_{r}(x)<s\right\}, \quad s \geq 0 .
$$

Hence, for $s \geq 0$, we have

$$
\begin{aligned}
\tau\left(\chi_{(s, \infty)}(|x-1|)\right)= & m\left\{r: 0 \leq r \leq \tau(1) \text { and } \mu_{r}(x)<1-s\right\} \\
& +m\left\{r \geq 0: \mu_{r}(x)>1+s\right\} \\
= & m\left\{r \geq 0:\left|\mu_{r}(x)-\mu_{r}(1)\right|>s\right\}
\end{aligned}
$$

consequently,

$$
\mu_{t}(x-1)=\mu_{t}(\mu(x)-\mu(1)) \text { for all } t \geq 0 .
$$

Lemma 2.2. If $e \in \mathscr{M}$ is a projection, $\tau(e)<\infty$, and $0 \leq x \in \widetilde{\mathscr{M}}$, then

$$
\mu(\text { exe }-e)=\mu(\mu(\text { exe })-\mu(e)) \text {. }
$$


Proof. For $y \in \mathscr{M}$, denote by $y_{e}$ the restriction of $e y$ to $e(H)$. Let $\mathscr{M}_{e}=$ $\left\{y_{e}: y \in \mathscr{M}\right\}$. Then $\mathscr{M}_{e}$ is a von Neumann algebra in the Hilbert space $e(H)$. Define $\tau_{e}$ on $\mathscr{M}_{e}$ by

$$
\tau_{e}\left(y_{e}\right)=\tau(\text { eye }), \quad y \in \mathscr{M} .
$$

$\tau_{e}$ is a finite faithful normal trace on $\mathscr{M}_{e}$. From Lemma 1 , with $\mathscr{M}, \tau$ replaced by $\mathscr{M}_{e}, \tau_{e}$, respectively, the result now follows.

While Lemma 2.2 suffices in what follows, we note the following strengthening of Lemma 2.1 .

Proposition 2.3. If $0 \leq x \in \widetilde{\mathscr{M}}_{0}$ then

$$
\mu(x-1)=\mu(\mu(x)-\mu(1)) .
$$

Proof. By Lemma 2.1, we may assume that $\tau(1)=\infty$, and since $0 \leq x \in \widetilde{\mathscr{M}}_{0}$, it follows that

$$
\tau\left(\chi_{[0, s)}(x)\right)=\infty \text { and } m\left\{r \geq 0: \mu_{r}(x)<s\right\}=\infty \text { for all } s>0 .
$$

If $0 \leq s<1$, then

$$
\tau\left(\chi_{(s, \infty)}(|x-1|)\right)=m\left\{r \geq 0:\left|\mu_{r}(x)-\mu_{r}(1)\right|>s\right\}=\infty .
$$

If $s \geq 1$, then

$$
\tau\left(\chi_{(s, \infty)}(|x-1|)\right)=\tau\left(\chi_{(1+s, \infty)}(x)\right)
$$

and

$$
m\left\{r \geq 0:\left|\mu_{r}(x)-\mu_{r}(1)\right|>s\right\}=m\left\{r \geq 0: \mu_{r}(x)>1+s\right\} .
$$

Consequently,

$$
\tau\left(\chi_{(s, \infty)}(|x-1|)\right)=m\left\{r \geq 0:\left|\mu_{r}(x)-\mu_{r}(1)\right|>s\right\} \quad \text { for all } s \geq 0,
$$

and the proposition is proved.

It is worth noting that the equality asserted by Proposition 2.3 may fail in general. In fact, let $\mathscr{M}$ be $L^{\infty}\left(\mathbb{R}^{+}\right)$, and let $x$ be given by setting

$$
x(t)=1-e^{-t}, \quad t \in \mathbb{R}^{+} .
$$

It is clear that $\mu(\mu(x)-\mu(1))=0$; on the other hand,

$$
\mu_{t}(x-1)=e^{-t}, \quad t \in \mathbb{R}^{+} \text {. }
$$

\section{A SINGULAR VAlUE INEQUALITY}

The main result of this paper now follows.

Theorem 3.1. If $0 \leq x \in \widetilde{\mathscr{M}}$ and $u \in \mathscr{M}$ is unitary then

$$
x-1 \nprec x-u \text {. }
$$

Proof. If $\tau(1)<\infty$, then Lemma 2.1 implies that

$$
\mu(x-1)=\mu(\mu(x)-\mu(1)),
$$

and since $\mu(u)=\mu(1)$, the general Markus inequality implies that

$$
\mu(x-1) \nprec \mu(x-u) \text {. }
$$


Assume then that $\tau(1)=\infty$. It suffices to show that, for any numbers $a>0, \varepsilon>0$, there exists $\tilde{x}=\tilde{x}(a, \varepsilon) \in \widetilde{\mathscr{M}}$ and a projection $e=e(a, \varepsilon) \in \mathscr{M}$ such that

(i) $\int_{0}^{a} \mu_{t}(x-1) d t=\int_{0}^{a} \mu_{t}(\mu(\tilde{x} e)-\mu(e)) d t$,

(ii) $\|\tilde{x} e-x e\|_{\infty} \leq \frac{\varepsilon}{a}$.

In fact, if this is done, then (ii) implies that

$$
\int_{0}^{a} \mu_{t}(\tilde{x} e-x e) d t \leq a\|\tilde{x} e-x e\|_{\infty} \leq \varepsilon
$$

and so, from (i) and the general Markus inequality, it follows that

$$
\begin{aligned}
\int_{0}^{a} \mu_{t}(x-1) d t & =\int_{0}^{a} \mu_{t}(\mu(\tilde{x} e)-\mu(e)) d t \\
& \leq \int_{0}^{a} \mu_{t}(\mu(\tilde{x} e)-\mu(x e)) d t+\int_{0}^{a} \mu_{t}(\mu(x e)-\mu(e)) d t \\
& \leq \int_{0}^{a} \mu_{t}(\tilde{x} e-x e) d t+\int_{0}^{a} \mu_{t}(\mu(x e)-\mu(u e)) d t \\
& \leq \varepsilon+\int_{0}^{a} \mu_{t}((x-u) e) d t \leq \varepsilon+\int_{0}^{a} \mu_{t}(x-u) d t
\end{aligned}
$$

We write

$$
\begin{aligned}
& \alpha=\inf \left\{s \geq 0: \tau\left(\chi_{(s, \infty)}(x)\right)<\infty\right\}, \\
& \beta=\sup \left\{s \geq 0: \tau\left(\chi_{[0, s)}(x)\right)<\infty\right\}, \\
& \gamma=\inf \left\{s \geq 0: \tau\left(\chi_{(s, \infty)}(|x-1|)\right)<\infty\right\} .
\end{aligned}
$$

Note that $\beta \leq \alpha$ since $\tau(1)=\infty$, and it is not difficult to see that

$$
\gamma=\max (|\alpha-1|,|1-\beta|)= \begin{cases}|\alpha-1|=\alpha-1 \quad \text { if } \frac{\alpha+\beta}{2} \geq 1, \\ |1-\beta|=1-\beta \quad \text { if } \frac{\alpha+\beta}{2} \leq 1\end{cases}
$$

We set $e^{\prime}=\chi_{(\gamma, \infty)}(|x-1|)$ and consider the following two cases.

Case 1. $0 \leq a \leq \tau\left(e^{\prime}\right)$. If $\tau\left(e^{\prime}\right)<\infty$, then we may take $\tilde{x}=x$ and $e=e^{\prime}$. Via the argument of [DDP2, Lemma 2.4(i)], observe that

$$
\chi_{[0, a)} \mu(x-1)=\chi_{[0, a)} \mu\left((x-1) e^{\prime}\right) .
$$

By Lemma 2.2, we now have

$$
\int_{0}^{a} \mu_{t}(x-1) d t=\int_{0}^{a} \mu_{t}\left((x-1) e^{\prime}\right) d t=\int_{0}^{a} \mu_{t}(\mu(e \tilde{x} e)-\mu(e)) d t .
$$

If $\tau\left(e^{\prime}\right)=\infty$, then there exists $s>\gamma$ such that

$$
a \leq \tau\left(\chi_{(s, \infty)}(|x-1|)\right)<\infty .
$$

Then we can take

$$
\tilde{x}=x \text { and } e=\chi_{(s, \infty)}(|x-1|),
$$

and it again follows that (i) is satisfied.

Case 2. $\tau\left(e^{\prime}\right)<a$. If $\gamma=0$ and $e_{1}=\chi_{\{1\}}(x)$, then $\tau\left(e_{1}\right)=\infty$ and $x e_{1}=e_{1}$. Let $e_{2} \in \mathscr{M}$ be a projection such that

$$
e_{2} \leq e_{1} \quad \text { and } \quad a-\tau\left(e^{\prime}\right) \leq \tau\left(e_{2}\right)<\infty .
$$


Note that $e_{2} \perp e^{\prime}$ and $x e_{2}=x e_{1} e_{2}=e_{2}$. If we take

$$
e=e^{\prime}+e_{2} \text { and } \tilde{x}=x e,
$$

then

$$
\tilde{x}=e x e=e \tilde{x} e \text { and } \tau(e)<\infty .
$$

From Lemma 2.2, it follows that

$$
\begin{aligned}
\int_{0}^{a} \mu_{t}(x-1) d t & =\int_{0}^{a} \mu_{t}\left((x-1) e^{\prime}\right) d t \\
& =\int_{0}^{a} \mu_{t}\left(\left(x e^{\prime}+x e_{2}\right)-\left(e^{\prime}+e_{2}\right)\right) d t \\
& =\int_{0}^{a} \mu_{t}(\mu(e \tilde{x} e)-\mu(e)) d t
\end{aligned}
$$

If $\gamma>0$, we may assume $\varepsilon$ satisfies $0<\varepsilon \leq 2 a \gamma$. Now define the projection $e_{1}$ via

$$
e_{1}= \begin{cases}\chi_{(\alpha-\varepsilon / a, \alpha]}(x) & \text { if } \gamma=|\alpha-1|, \\ \chi_{[\beta, \beta+\varepsilon / a)}(x) & \text { if } \gamma=|1-\beta| .\end{cases}
$$

It follows that $\tau\left(e_{1}\right)=\infty$ and $e_{1} \perp e^{\prime}$. Let $e_{2} \in \mathscr{M}$ be a projection such that

$$
e_{2} \leq e_{1} \text { and } a-\tau\left(e^{\prime}\right) \leq \tau\left(e_{2}\right)<\infty .
$$

Define $e=e^{\prime}+e_{2}$ and

$$
\tilde{x}= \begin{cases}x e^{\prime}+\alpha e_{2} & \text { if } \gamma=|\alpha-1| \\ x e^{\prime}+\beta e_{2} & \text { if } \gamma=|1-\beta|\end{cases}
$$

It follows that

$$
\tilde{x}-x e= \begin{cases}(\alpha-x) e_{2} & \text { if } \gamma=|\alpha-1|, \\ (\beta-x) e_{2} & \text { if } \gamma=|1-\beta|,\end{cases}
$$

and consequently (ii) is satisfied. Moreover,

$$
|\tilde{x}-e|= \begin{cases}|x-1| e^{\prime}+|\alpha-1| e_{2} & \text { if } \gamma=|\alpha-1|, \\ |x-1| e^{\prime}+|1-\beta| e_{2} & \text { if } \gamma=|1-\beta|,\end{cases}
$$

which implies that

$$
|\tilde{x}-e|=|x-1| e^{\prime}+\gamma e_{2},
$$

and hence

$$
\begin{aligned}
\mu(\tilde{x}-e) & =\mu\left(|x-1| e^{\prime}+\gamma e_{2}\right) \\
& =\mu\left(|x-1| e^{\prime}\right)+\gamma \chi_{\left[\tau\left(e^{\prime}\right), \tau\left(e^{\prime}\right)+\tau\left(e_{2}\right)\right)} \\
& =\mu(x-1) \chi_{[0, \tau(e))} .
\end{aligned}
$$

As $e \tilde{x}=\tilde{x} e=\tilde{x}$ and $a \leq \tau(e)<\infty$, it follows again via Lemma 2.2 that (i) is satisfied, and consequently the proof of the theorem is complete.

We remark that Theorem 3.1 is due, in the case of $n \times n$ matrices, to Fan and Hoffman [FH] and to van Riemsdijk [vR] for the case that $\mathscr{M}=L(H)$ equipped with standard trace and $x$ is bounded. The present approach is based on that of van Riemsdijk.

Let $L^{0}\left(\mathbb{R}^{+}\right)$be the linear space of all (equivalence classes of) complex-valued Lebesgue measurable functions on the halfline $\mathbb{R}^{+}$. A Banach space $E\left(\mathbb{R}^{+}\right)$with norm $\|.\|_{E}$, which is a linear subspace of $L^{0}\left(\mathbb{R}^{+}\right)$, is called a fully symmetric 
Banach function space on $\mathbb{R}^{+}$if and only if $f \in L^{0}\left(\mathbb{R}^{+}\right), g \in E\left(\mathbb{R}^{+}\right)$and $\mu(f) \preccurlyeq \mu(g)$ imply that $f \in E\left(\mathbb{R}^{+}\right)$and $\|f\|_{E} \leq\|g\|_{E}$. It is shown in [KPS, Theorem II 4.3] that the fully symmetric Banach function spaces on $\mathbb{R}^{+}$are precisely the exact interpolation spaces for the Banach couple $\left(L^{1}\left(\mathbb{R}^{+}\right), L^{\infty}\left(\mathbb{R}^{+}\right)\right)$.

If $E\left(\mathbb{R}^{+}\right)$is a fully symmetric Banach function space, we define

$$
E(\mathscr{M})=\{x \in \widetilde{\mathscr{M}}: \mu(x) \in E\}
$$

and set

$$
\|x\|_{E(\mathscr{R})}=\|\mu(x)\|_{E}, \quad x \in E(\mathscr{M}) .
$$

It can be shown (see [DDP1, DDP2]) that $\left(E(\mathscr{M}),\|\cdot\|_{E(\mathscr{K})}\right)$ is a Banach space. Of course, each of the familiar spaces $L^{p}\left(\mathbb{R}^{+}\right), 1 \leq p \leq \infty$, is fully symmetric. In this case, the corresponding noncommutative spaces $L^{p}(\mathscr{M}), 1 \leq p \leq \infty$, are precisely the spaces considered by Nelson [Ne] and reduce to the familiar Schatten $p$-classes in the special case that $\mathscr{M}$ is $L(H)$ equipped with standard trace.

The following result, which extends the metric inequalities given in [FH, GK, $\mathrm{vR}, \mathrm{AEG}]$, is now an immediate consequence of Theorem 3.1 and the preceding definitions.

Corollary 3.2. Let $E\left(\mathbb{R}^{+}\right)$be a fully symmetric Banach function space. If $0 \leq$ $x \in \widetilde{\mathscr{M}}, u$ is unitary, and $x-u \in E(\mathscr{M})$, then $x-1 \in E(\mathscr{M})$ and

$$
\|x-1\|_{E(\mathscr{A})} \leq\|x-u\|_{E(\mathscr{A})} .
$$

We note finally that equality in Theorem 3.1, and hence also in Corollary 3.2 , may hold for some unitary $u \neq 1$. In fact, if $\mathscr{M}$ is the von Neumann algebra $L^{\infty}[0,1]$, acting by multiplication on $L^{2}[0,1]$, set $x=\chi_{\left[0, \frac{1}{2}\right]}$ and $u=\chi_{[0,1 / 2]}-\chi_{[1 / 2,1]}$. Then it is easily verified that

$$
\mu(x-1)=\mu(x-u) .
$$

\section{UNIQUENESS OF BEST APPROXIMATION}

The corollary of $\S 3$ shows that if $0 \leq x \in \widetilde{\mathscr{M}}$, then 1 is a best unitary approximant to $x$ in any fully symmetric norm, and as noted in the final example, unless further restrictions are imposed, then $x$ need not have a unique best approximant for any fully symmetric norm. Following the terminology of [AEG], if $0 \leq x \in \widetilde{\mathscr{M}}$, then $x$ will be called strictly positive if $\operatorname{ker} x=\{0\}$. We will now show that, if $x$ is strictly positive and $x-1 \in L^{p}(\mathscr{M})$ for some $p, 1<p<\infty$, then 1 is necessarily the unique best unitary approximant to $x$ in $L^{p}(\mathscr{M})$. This extends a similar result obtained in [AEG] for the special case of the Schatten $p$-classes, and our method here is a suitable adaptation of the approach of [AEG].

It is well known (see, for example [FK, Theorems 5.2, 5.3]) that the noncommutative spaces $L^{p}(\mathscr{M}), 1<p<\infty$, satisfy the Clarkson-McCarthy inequalities, and hence are uniformly convex. Consequently, from the known duality theory for the $L^{p}(\mathscr{M})$-spaces [Ne, DDP3], it follows that $L^{p}(\mathscr{M}), 1<p<\infty$, is uniformly smooth and has uniformly Fréchet-differentiable norm. (See [Be, 3 II 2 Propositions 1, 2].) If $0 \neq x \in L^{p}(\mathscr{M}), 1<p<\infty$, and we set

$$
G_{x}(h)=\lim _{t \rightarrow 0} \frac{\|x+t h\|_{p}-\|x\|_{p}}{t}, \quad h \in L^{p}(\mathscr{M}),
$$


then $G_{x}$ is the unique real linear functional defined on $L^{p}(\mathscr{M})$ satisfying

$$
\left\|G_{x}\right\|=1 \text { and } G_{x}(x)=\|x\|_{p} .
$$

These remarks constitute the proof of the first part of the following lemma.

Lemma 4.1. If $1<p<\infty$, then $L^{p}(\mathscr{M})$ has Fréchet-differentiable norm, and if $0 \neq x \in L^{p}(\mathscr{M})$, then

$$
G_{x}(h)=\operatorname{Re} \tau\left(\left(\frac{|x|}{\|x\|_{p}}\right)^{p-1} w^{*} h\right), \quad h \in L^{p}(\mathscr{M}),
$$

where $x=w|x|$ is the polar decomposition of $x$.

Proof. It remains only to establish the formula for the derivative. If we set

$$
F_{x}(h)=\operatorname{Re} \tau\left(\left(\frac{|x|}{\|x\|_{p}}\right)^{p-1} w^{*} h\right), \quad h \in L^{p}(\mathscr{M}),
$$

then it is clear that $F_{x}$ is a real linear functional on $L^{p}(\mathscr{M})$ for which $F_{x}(x)=$ $\|x\|_{p}$. It suffices to show that $\left\|F_{x}\right\|=1$. This follows from the usual Minkowski inequality by noting that $\left(|x| /\|x\|_{p}\right)^{p-1} \in L^{q}(\mathscr{M})$, with $1 / p+1 / q=1$, and that

$$
\begin{aligned}
\left|F_{x}(h)\right| & \leq\left|\tau\left(\left(|x| /\|x\|_{p}\right)^{p-1} w^{*} h\right)\right| \\
& \leq\left\|\left(|x| /\|x\|_{p}\right)^{p-1}\right\|_{q}\left\|w^{*} h\right\|_{p} \\
& \leq\|h\|_{p} \quad \text { for all } h \in L^{p}(\mathscr{M}) .
\end{aligned}
$$

We may now state the principal result of this section, which extends [AEG, Theorem 3.2, 3.5].

Theorem 4.2. If $x \in \widetilde{\mathscr{M}}$ is strictly positive and there exists a unitary operator $u$ such that $x-u \in L^{p}(\mathscr{M}), 1<p<\infty$, then it follows that $x-1 \in L^{p}(\mathscr{M})$ and

$$
\|x-1\|_{p}<\|x-u\|_{p} \quad \text { for all unitary } u \neq 1 \text {. }
$$

Proof. Corollary 3.2 and the assumption of the theorem imply that $x-1 \in$ $L^{p}(\mathscr{M})$ and

$$
\|x-1\|_{p} \leq\|x-u\|_{p} \quad \text { for all unitary } u .
$$

It remains to show that if $v$ is unitary and

$$
\|x-v\|_{p} \leq\|x-u\|_{p} \quad \text { for all unitary } u,
$$

then $v=1$. To do this, we first show that such an operator $v$ must be selfadjoint and commute with $x$. We may assume that $x-v \neq 0$. If $f \in \mathscr{M}$ is a projection with $\tau(f)<\infty$, we define, following [AEG],

$$
u_{f}(\theta)=e^{i \theta} f+(1-f), \quad \theta \in \mathbb{R} .
$$

It is clear that $u_{f}(\theta)$ is unitary. For each $f$, the composition of maps

$$
\theta \rightarrow x-v u_{f}(\theta) \rightarrow\left\|x-v u_{f}(\theta)\right\|_{p}, \quad \theta \in \mathbb{R},
$$

has a local minimum at $\theta=0$. It follows from Lemma 4.1 and the chain rule that

$$
\left.\frac{d}{d \theta}\left\|x-v u_{f}(\theta)\right\|_{p}\right|_{\theta=0}=\operatorname{Re} \tau\left(\left(\frac{|x-v|}{\|x-v\|_{p}}\right)^{p-1} w^{*}(-i v f)\right)
$$


where $x-v=w|x-v|$ is the polar decomposition of $x-v$. Consequently,

$$
\tau\left(\left(|x-v|^{p-1} w^{*} v-v^{*} w|x-v|^{p-1}\right) f\right)=0
$$

for all projections $f \in \mathscr{M}$ with $\tau(f)<\infty$. Since linear combinations of projections of finite trace are dense in $L^{p}(\mathscr{M})$, it follows that

$$
|x-v|^{p-1} w^{*} v=v^{*} w|x-v|^{p-1},
$$

and so $v^{*} w|x-v|^{p-1}$ is selfadjoint. The same argument as in Lemma 3.3 of [AEG] now shows that $v$ is selfadjoint and commutes with $x$.

From (1) and (2), it follows that

$$
\|x-1\|_{p}=\|x-v\|_{p} .
$$

Setting $e=(1-v) / 2$, note that $e$ is a selfadjoint projection commuting with $x$ and that $x e \geq 0$. From (3),

$$
\int_{[0, \infty)} \mu_{t}^{p}(x-1) d t=\int_{[0, \infty)} \mu_{t}^{p}\left(\sqrt{(x-1)^{2}+4 x e}\right) d t
$$

and since $|x-1| \leq \sqrt{(x-1)^{2}+4 x e}$, it follows that

and so

$$
\mu(x-1)=\mu\left(\sqrt{(x-1)^{2}+4 x e}\right),
$$

Now, for all $s>0$,

$$
\mu\left((x-1)^{2}\right)=\mu\left((x-1)^{2}+4 x e\right) .
$$

$$
\begin{aligned}
\tau\left(\chi_{(s, \infty)}\left((x-1)^{2}\right)\right)= & \tau\left(\chi_{(s, \infty)}\left((x-1)^{2}\right) e\right)+\tau\left(\chi_{(s, \infty)}\left((x-1)^{2}\right)(1-e)\right) \\
= & \tau\left(\chi_{(s, \infty)}\left((x-1)^{2} e\right)\right)+\tau\left(\chi_{(s, \infty)}\left((x-1)^{2}(1-e)\right)\right), \\
\tau\left(\chi_{(s, \infty)}\left((x-1)^{2}+4 x e\right)\right)= & \tau\left(\chi_{(s, \infty)}\left((x-1)^{2}+4 x e\right) e\right) \\
& +\tau\left(\chi_{(s, \infty)}\left((x-1)^{2}+4 x e\right)(1-e)\right) \\
= & \tau\left(\chi_{(s, \infty)}\left((x+1)^{2} e\right)\right)+\tau\left(\chi_{(s, \infty)}\left((x-1)^{2}(1-e)\right)\right),
\end{aligned}
$$

since $x-1 \in L^{p}(\mathscr{M})$ implies that $\tau\left(\chi_{(s, \infty)}\left((x-1)^{2}\right)<\infty\right.$ for all $s>0$; hence

$$
\mu\left((x-1)^{2} e\right)=\mu\left((x+1)^{2} e\right),
$$

and so

$$
\mu^{p}(|x-1| e)=\mu^{p / 2}\left((x-1)^{2} e\right)=\mu^{p}((x+1) e) .
$$

Thus

$$
\tau\left(|x-1|^{p} e\right)=\tau\left((x+1)^{p} e\right)<\infty,
$$

and since

$$
|x-1|^{p} e \leq(x+1)^{p} e,
$$

it follows from faithfulness of $\tau$ that

$$
|x-1|^{p} e=(x+1)^{p} e,
$$

and so

It now follows that

$$
|x-1| e=(x+1) e .
$$

and this implies that

$$
(x-1)^{2} e=(x+1)^{2} e,
$$

$$
x e=0 .
$$

As $\operatorname{ker} x=\{0\}$, this implies that $e=0$. 


\section{REFERENCES}

[AEG] J. G. Aiken, J. A. Erdos, and J. A. Goldstein, Unitary approximation of positive operators, Illinois J. Math. 24 (1980), 61-72.

[Be] B. Beauzamy, Introduction to Banach spaces and their geometry, North-Holland Math. Stud., vol. 68, North-Holland, Amsterdam and New York, 1982.

[DDP1] P. G. Dodds, T. K. Dodds, and B. de Pagter, A general Markus inequality, Proc. Centre Math. Anal. Austral. Nat. Univ., vol. 24, Austral. Nat. Univ., Canberra, 1989, pp. 47-57.

[DDP2] _ , Non-commutative Banach function spaces, Math. Z. 201 (1989), 583-597.

[DDP3] _ Non-commutative Köthe duality, Trans. Amer. Math. Soc. (to appear).

[FH] Ky Fan and A. J. Hoffman, Some metric inequalities in the space of matrices, Proc. Amer. Math. Soc. 6 (1955), 111-116.

[FK] T. Fack and H. Kosaki, Generalized s-numbers of $\tau$-measurable operators, Pacific J. Math. 123 (1986), 269-300.

[GK] I. C. Gohberg and M. G. Krein, Introduction to the theory of linear non-selfadjoint operators, Transl. Math. Monographs, vol. 18, Amer. Math. Soc., Providence, RI, 1969.

[KPS] S. G. Kreĭn, Ju. I. Petunin, and E. M. Semenov, Interpolation of linear operators, Transl. Math. Monographs, vol. 54, Amer. Math. Soc., Providence, RI, 1982.

[Li] V. B. Lidskii, On the eigenvalues of sums and products of symmetric matrices, Dokl. Akad. Nauk SSSR 75 (1950), 769-772. (Russian)

[Ma] A. S. Markus, The eigen- and singular values of the sum and product of linear operators, Russian Math. Surveys 19 (1964), 91-120.

[Ne] E. Nelson, Notes on non-commutative integration, J. Funct. Anal. 15 (1974), 103-116.

[vR] D. J. van Riemsdijk, Some metric inequalities in the space of bounded linear operators on a separable Hilbert space, Nieuw Arch. Wisk. (4) 20 (1972), 216-230.

[Wi] H. Wielandt, An extremum property of sums of eigenvalues, Proc. Amer. Math. Soc. 6 (1955), 106-110.

School of Information Science and Technology, The Flinders University of South Australia, GPO Box 2100, Adelaide 5001, Australia 